\title{
Diabetes Technology and the Human Factor
}

\author{
Alon Liberman ${ }^{1}$ and Katharine Barnard ${ }^{2}$
}

\section{Introduction}

Undoubtedly, Diabetes technologies have revolutionized the way that glycemic control is optimized for many people with diabetes, particularly type 1 diabetes. Advances in recent years reflect something of a revolution in diabetes management with the promise of further developments. Advances have moved beyond engineering excellence and biomedical impact to greater understanding and emphasis on the psychosocial impact of such technologies. The ability or willingness to incorporate a technology into one's everyday life is the ultimate test of success.

For this year's article on diabetes technology and the human factor, we have reviewed a range of technologies future, new, and established in the context of their impact on the lives of people with diabetes. We do this from the perspective of human factors important to successful uptake and continued use of technologies to ensure the reduced burden of diabetes and optimization of glycemic control and quality of life. Acceptability, usability, device burden and benefits, visibility, and smooth integration into everyday living are all important factors that must be accounted for when considering the introduction of new technology.

The aim of this article is to review some of the most prominent articles published over the past year addressing the issue of the human factor in the use of diabetes technologies. The criteria for article selection were human factor research regarding advanced technological devices, with particular focus on studies that have examined ways to assist populations with special and often challenging needs, such as children and older people, in order to make optimal use of diabetes technologies. Finally, our included studies pay attention to the experience of users alongside the point of view of physicians and engineers.

Included technologies cover continuous glucose monitoring systems, both externally worn and a novel implantable device, a bolus calculator in self-monitoring of blood glucose device, remote monitoring of continuous glucose monitoring (CGM), and artificial pancreas systems. These represent a broad range of technologies currently available and coming to market in the near future. While novel technologies remain of great interest, it is important to consider existing technologies and how they can best be used to improve outcomes for people with diabetes, which sadly remain stubbornly suboptimal for many. We look forward with interest and optimism as the human factors associated with diabetes technologies continue to demand their place as central to success.

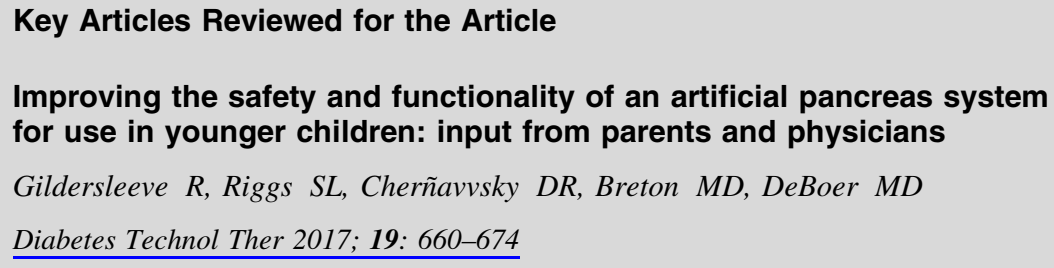

${ }^{1}$ Jesse Z and Lea Sara Shafer Institute of Endocrinology and Diabetes, National Center for Childhood Diabetes, Schneider Children's Medical Center of Israel, Petah Tikva, Israel

${ }^{2}$ Faculty of Health and Social Sciences, Bournemouth University, Bournemouth, UK 
Acceptability of implantable continuous glucose monitoring sensor

Barnard KD, Kropff J, Choudhary P, Neupane S, Bain SC, Kapitza C, Forst T, Link $M$, Mdingi $C$, DeVries JH

J Diabetes Sci Technol 2018; 12: 634-638

Behavioral patterns and associations with glucose control during 12-week randomized free-living clinical trial of day and night hybrid closed-loop insulin delivery in adults with type 1 diabetes

Emami A, Willinska ME, Thabit H, Leelarathna L, Hartnell S, Dellweg S, Benesch C, Mader JK, Holzer M, Kojzar H, Pieber TR, Arnolds S, Evans ML, Hovorka $R$

Diabetes Technol Ther 2017; 19: 433-437

Bolus calculator reduces hypoglycemia in the short term and fear of hypoglycemia in the long term in subjects with type 1 diabetes (CBMDI study)

Vallejo Mora MDR, Carreira M, Anarte MT, Linares $F$, Olveira G, González Romero $S$

Diabetes Technol Ther 2017; 19: 402-409

First experiences with a wearable multisensor in an outpatient glucose monitoring study, part I: the users' view

Caduff A, Zanon M, Zakharov P, Mueller M, Talary M, Krebs A, Stahel WA, Donath M J Diabetes Sci Technol 2018; 12: 562-568

First experiences with a wearable multisensor device in a noninvasive continuous glucose monitoring study at home, part II: the investigators' view

Zanon M, Mueller M, Zakharov P, Talary MS, Donath M, Stahel WA, Caduff A

J Diabetes Sci Technol 2018; 12: 554-561

In-home nighttime predictive low glucose suspend experience in children and adults with type 1 diabetes

Messer LH, Calhoun P, Buckingham B, Wilson DM, Hramiak I, Ly TT, Driscoll M, Clinton P, Maahs DM; for the In Home Closed Loop Study Group

Pediatr Diabetes 2017; 18: 332-339

Optimizing hybrid closed-loop therapy in adolescents and emerging adults using the MiniMed 670G system

Messer LH, Forlenza GP, Sherr JL, Wadwa RP, Buckingham BA, Weinzimer SA, Maahs DM, Slover RH

Diabetes Care 2018; 41: 789-796

Real-time continuous glucose monitoring facilitates feelings of safety in older adults with type 1 diabetes: a qualitative study

Litchman ML, Allen NA

J Diabetes Sci Technol 2017; 11: 988-995

Schooling diabetes: Use of continuous glucose monitoring and remote monitors in the home and school settings

Erie C, Van Name MA, Weyman K, Weinzimer SA, Finnegan J, Sikes K,

Tamborlane $W V$, Sherr JL

Pediatr Diabetes 2018; 19: 92-97

Continuous glucose monitoring in patients with type 1 diabetes and impaired awareness of hypoglycemia: also effective in patients with psychological distress?

van Beers CAJ, de Wit M, Kleijer SJ, Geelhoed-Duijvestijn PH, DeVries JH, Kramer MHH, Diamant M, Serné EH, Snoek FJ

Diabetes Technol Ther 2017; 19: 595-599 
Improving the safety and functionality of an artificial pancreas system for use in younger children: input from parents and physicians

Gildersleeve $R^{1}$, Riggs $S L^{2}$, Cherñavsky $D R^{1,3}$, Breton $M D^{1,3}$, DeBoer $M D^{1,4}$

${ }^{1}$ Center for Diabetes Technology, University of Virginia, Charlottesville, VA; ${ }^{2}$ Department of Industrial Engineering, Clemson University, Clemson, SC; ${ }^{3}$ TypeZero Technologies, Inc., Charlottesville, VA; ${ }^{4}$ Department of Pediatrics, Division of Pediatric Endocrinology, University of Virginia, Charlottesville, VA

Diabetes Technol Ther 2017; 19: 660-674

\section{Background}

Artificial pancreas (AP) systems have initially been designed for-and examined in-adolescents and adults, yet there is evidence that an AP system with additional support and safety features could considerably benefit younger patients with type 1 diabetes (T1D).

\section{Subjects and Methods}

Five pediatric endocrinologists along with 15 parents of young patients (5-8 years of age) with T1D took part in four focus groups. Focus groups explored current diabetes utilization and acceptance of technology in addition to potential modifications to the current adult AP system, which would enable younger children to use the technology in a safe and successful manner. Child-specific input task functionality, safety features, and monitoring capabilities were discussed.

\section{Results}

Participants' suggestions included passcode entry for parents, ancillary caregivers, and the child, allowing control over access to each AP feature; preset categories for early, intermediate, and advanced child access; maximum level of customization for general and alarm settings; easy-tounderstand meal screens that employ the AP's corrective blood glucose (BG) ability; automated exercise mode; voice command and spoken messaging capabilities; emergency contacts; treatment instructions for both the young patient and the caregiver; website and application for remote monitoring; animated CGM BG trace; gamification, such as reinforcing diabetes-friendly behaviors; and comprehensive training for all those involved in managing the child's diabetes.

\section{Conclusion}

Both the parents and the endocrinologists who participated in the focus groups expressed enthusiasm for AP applications accessible to younger children with T1D. They also emphasized that an adjusted system could better serve this group's needs for safety and improved diabetes-related communication. The diverse and developing needs of 5- to 8-year-old patients require adaptable and adjustable systems for T1D management.

\section{Comments}

This study continues with the welcome trend of collecting information about the effectiveness of using diabetes technology (in this case, artificial pancreatic systems) from users as well as physicians and engineers. Artificial pancreatic systems have been designed for use by adults and adolescents. Their suitability for use by children poses several challenges. The authors note several risk factors, including inadequate oversight, understanding, and training and purposeful misuse, which significantly contribute to reported adverse events with pediatric use of insulin pumps $(1,2)$. Among the research recommendations that came up, it is interesting to note that participants supported simple, written, and pictorial instructions accompanying alarms to help children and other care providers with treatment decisions and alleviate alarm-induced anxiety among school staff. In addition, parents suggested development of a modified and flexible AP system to help children assume more responsibility for their diabetes management and creation of a system that can facilitate the involvement of and communication among various care providers. It will be interesting to see how closely the views of children, their parents, and health-care providers align in terms of their perceived needs and desires of the technology in future research.

\section{Acceptability of implantable continuous glucose monitoring sensor}

Barnard $K D^{1,2}$, Kropff $J^{3}$, Choudhary $P^{4}$, Neupane $S^{5}$, Bain $S C^{6}$, Kapitza $C^{7}$, Forst $T^{8}$, Link $M^{9}$, Mdingi $C^{10}$, DeVries $\mathrm{JH}^{3}$

${ }^{1}$ Faculty of Health and Social Sciences, Bournemouth University, Bournemouth, UK; ${ }^{2}$ BHR Limited, Fareham, UK; ${ }^{3}$ Department of Endocrinology, Academic Medical Centre, University of Amsterdam, Amsterdam, the Netherlands; ${ }^{4}$ King's College London, London, UK; ${ }^{5}$ Cambridge University Hospitals NHS Foundation Trust, Cambridge, UK; ${ }^{6}$ Joint Clinical Research Facility, Swansea University, Swansea, UK; ${ }^{7}$ Profil, Neuss, Germany; ${ }^{8}$ Profil, Mainz, Germany; 9 Institut für Diabetes-Technologie Forschungsund Entwicklungsgesellschaft $\mathrm{mbH}$ an der Universität Ulm, Ulm, Germany; ${ }^{10}$ Research, Senseonics Inc, Germantown, $M D$

J Diabetes Sci Technol 2018; 12: 634-638

\section{Background}

Continuous glucose monitoring has been shown to enhance diabetes care. However, there are some barriers related to the device visibility, psychological functioning, and sensor durability. Implantable sensors may be of help in coping with these challenges.

\section{Methods}

The PRECISE trial was conducted with the aim of evaluating the acceptability of implantable CGMs. This evaluation was done by means of questionnaires given to study subjects that included the Diabetes Distress Scale, the CGM impact scale, and bespoke device satisfaction. 


\section{Results}

Fifty-one subjects-10 from the UK and 41 from Germany-completed the surveys: $90 \%$ had T1D, 50\% were treated with insulin pumps, and $45 \%$ were past CGM users. In results from the CGM Impact Scale, $86 \%(n=44)$ of subjects reported that they felt better about their diabetes control, while $14 \%$ felt neutral. Ninety percent of CGM-naïve subjects and $81 \%$ past CGM users reported an increase in confidence regarding their diabetes treatment. In addition, $73 \%$ $(n=37)$ felt safer while sleeping and $78 \%(n=39)$ felt more confident about avoiding serious hypoglycemia (with $27 \%$ and $22 \%$, respectively, feeling neutral). Respondents showed an improvement in glycated hemoglobin (HbA1c), from 7.51 to 7.05 ( $P<0.0001)$ over 90 days of CGM use. In general, the system received high ratings on ease of use, convenience, and comfort. Overall, $84 \%$ of participants would choose to be implanted again, and $93 \%$ of CGM naïve participants, along with $86 \%$ past CGM users, reported that the burden of diabetes was decreased.

\section{Conclusion}

Implantable CGM devices were viewed favorably by users: A substantial majority of subjects (93\% of first-time users and $77 \%$ past CGM users) would choose to continue using the system for more efficient management of their diabetes.

\section{Comments}

This article reflects the growing acceptance of continuous glucose monitoring as part of type diabetes management and the diversity of both systems and user preferences. As systems become increasingly accurate, with fewer demands on the user in terms of calibration and feature personalization, choice moves on to human factor decisions in terms of visibility of diabetes, frequency of sensor change, and integration into other systems such as artificial pancreas systems. The ability to view data remotely, via smartphone or remote device, is increasingly attractive to users as it enables discreet diabetes management. Users will need to balance tradeoffs in terms of the implant procedure against longer sensor duration and in terms of loss of data when choosing not to wear the transmitter. Notwithstanding, the data reported here demonstrates reduced diabetes distress, high acceptance, and increased confidence regarding feeling safe when sleeping, all of which are crucial to minimizing the burden of diabetes whilst optimizing glycemic control.

\section{Behavioral patterns and associations with glucose control during 12-week randomized free-living clinical trial of day and night hybrid closed-loop insulin delivery in adults with type 1 diabetes}

Emami $A^{1}$, Willinska $M E^{1,2}$, Thabit $H^{1,3}$, Leelarathna $L^{1,4}$, Hartnell $S^{3}$, Dellweg $S^{5}$, Benesch $C^{5}$, Mader $J K^{6}$, Holzer $M^{6}$, Kojzar $H^{6}$, Pieber $T R^{6}$, Arnolds $S^{5}$, Evans $M L^{1,3}$, Hovorka $R^{1,2}$

${ }^{1}$ Wellcome Trust-MRC Institute of Metabolic Science, University of Cambridge, Cambridge, UK; ${ }^{2}$ Department of
Paediatrics, University of Cambridge, Cambridge, UK; ${ }^{3}$ Department of Diabetes \& Endocrinology, Cambridge University Hospitals NHS Foundation Trust, Cambridge, UK; ${ }^{4}$ Central Manchester University Hospitals NHS Foundation Trust and University of Manchester, Manchester, UK; ${ }^{5}$ Profil, Neuss, Germany; ${ }^{6}$ Division of Endocrinology and Diabetology, Department of Internal Medicine, Medical University of Graz, Graz, Austria

Diabetes Technol Ther 2017; 19: 433-437

\section{Objective}

Patterns of meal intake, insulin bolus delivery, and fingerstick glucose measurements during hybrid closed-loop and sensor-augmented pump (SAP) therapy were assessed, including correlations with glucose control.

\section{Methods}

Pump-treated adults with type 1 diabetes underwent, in random order, 12 weeks each of free-living closed-loop $(n=32)$ and 12 weeks SAP $(n=33)$ therapy, and results were retrospectively analyzed. Daily patterns of main meals, snacks, prandial insulin boluses, correction boluses, and fingerstick glucose measurements were quantified by analyzing information gathered on the research glucometer and research insulin pump.

\section{Results}

Overall, 1942 closed-loop days and 2530 SAP days were evaluated. During periods of closed-loop vs SAP therapy, the number of insulin boluses decreased by 1.0 per day (mean value [95\% confidence interval $0.6-1.4] ; P<0.001$ ), mainly due to a 0.7 per day (mean) reduction in number of correction boluses ([95\% CI 0.4-1.0]; $P<0.001$ ). Other behavioral patterns remained unchanged. The carbohydrate content, but not the number, of snacks was positively associated with glycemic variability as measured by standard deviation of sensor glucose (closed-loop $P<0.05$; SAP $P<0.01$ ), as well as mean sensor glucose $(P<0.05)$ and postintervention HbA1c $(P<0.05)$. Behavioral patterns explained $47 \%$ of between-subject variance in glucose variability during SAP period and $30 \%-33 \%$ of variance in mean sensor glucose and postintervention $\mathrm{HbA1c}$.

\section{Conclusion}

During periods of closed-loop therapy, fewer correction boluses were delivered. The size of snacks appears to cause a decline in glucose control, possibly owing to the carbohydrate-rich nature of the snacks. The results indicate that modifiable behavioral patterns might be essential factors in glucose control.

\section{Comments}

Diabetes management today relies heavily on patient behavior, and technologies should be developed to support the individuality of diabetes self-management rather than expecting behavior to fit the technology. The introduction of artificial pancreas systems does not alter 
this fact (as long as the artificial pancreas systems have not been fully automated). Understanding and adapting to users' eating habits, blood glucose monitoring, and bolus correction rates are crucial to achieving improved glycemic control. The results of the current study demonstrate that the number of correction boluses decreases during closed-loop period, reducing the total number of insulin boluses administered per day compared with sensor-augmented pump therapy. However, all other behavioral patterns were unchanged. The authors conclude that modifiable behavioral patterns may be essential requisites of glucose control, but this is surely the opposite of the expectations of AP systems by users. AP systems must be adaptable to the individual; otherwise, uptake and continued use will be limited to the few who are willing to meet the demands of the system as compared with the many who seek relief from the burden of diabetes.

\section{Bolus calculator reduces hypoglycemia in the short term and fear of hypoglycemia in the long term in subjects with type 1 diabetes (CBMDI study)}

Vallejo Mora $M D R^{1,2}$, Carreira $M^{2,3}$, Anarte $M T^{2,3}$, Linares $F^{2,4}$, Olveira $G^{1,2,4}$, González Romero $S^{1,2,4}$

${ }^{1}$ Endocrinology and Nutrition Department, Hospital Regional Universitario de Málaga, Málaga, Spain; ${ }^{2}$ Instituto de Investigación Biomédica, Hospital Regional Universitario de Málaga, Málaga, Spain; ${ }^{3}$ Personality, Evaluation and Psychological Treatment, School of Psychology, Málaga, Spain; ${ }^{4}$ CIBER of Diabetes and Metabolic Diseases, Barcelona, Spain

Diabetes Technol Ther 2017; 19: 402-409

\section{Background}

Previous research by the authors showed that patients with type 1 diabetes on multiple daily injections experienced increased metabolic control and decreased hypoglycemia after utilizing a bolus calculator for 4 months.

\section{Objective}

The current study was carried out to determine whether (1) extending the use of the bolus calculator or (2) introducing it in the control group, which had already been subjected to treatment intensification, could further improve metabolic control and related psychological issues.

\section{Methods}

After the previous 4-month clinical trial, in which the participants were randomly assigned to either the treatment group using the bolus calculator or the control group, both groups used the calculator during an additional 4-month period.

\section{Results}

The control group from the previous study did not show improvement in HbA1c after utilizing the device (HbAlc
$7.86 \% \pm 0.87 \%$ vs $8.01 \% \pm 0.93 \% ; P=0.215)$, despite the fact that a significant reduction in postprandial hypoglycemia was observed $(2.3 \pm 2$ vs $1.1 \pm 1.2 / 2$ weeks; $P=0.002)$. The treatment group, for which use of the calcualtor was extended from 4 to 8 months, did not show improvement in HbAlc either $(7.61 \pm 0.58$ vs $7.73 \pm 0.65 ; P=0.209)$. This original treatment group did, however, have greater perceived treatment satisfaction $(12.03 \pm 4.26$ vs $13.71 \pm 3.75 ; P=0.007)$ and significantly decreased fear of hypoglycemia $(28.24 \pm$ 8.18 basal vs $25.66 \pm 8.02$ at 8 months; $P=0.026$ ).

\section{Conclusions}

Extending the utilization of the bolus calculator or introducing it in a previously intensified control group did not result in improved metabolic control, although it did confirm a reduction in hypoglycemic episodes in the short term, whereas extending its use from 4 to 8 months correlated with increased treatment satisfaction and reduced fear of hypoglycemia.

\section{Comments}

Despite advances in developing new medications, insulin delivery systems, and glucose monitoring technology, a significant percentage of people with diabetes remain well above their glycemic goals. Because diabetes is largely a self-managed disease with a significant psychosocial burden for patients and their families, many people with diabetes experience poorer quality of life than those without diabetes. The burden of diabetes self-management may partly explain why many people with diabetes are unable to reach optimal glycemic targets; however, another compounding factor may be an underlying fear of hypoglycemia. Fear of hypoglycemia can be a key obstacle to intensifying therapy and/or adhering to prescribed insulin regimens, which can lead to poorer metabolic control and subsequent health outcomes. The bolus calculator's contribution to improved metabolic control and its impact on psychological issues such as fear of hypoglycemia and level of treatment satisfaction is documented in recent publications $(3,4,5)$. The authors found that participants who used the bolus calculator during the entire extended study saw a significant decrease in the fear of hypoglycemia as documented after a period of 8 months compared with the basal situation. It seems that when users feel confident and able to trust the device, they use it in many decisionmaking processes in a way that positively affects their self-management and improves their quality of life.

\section{First experiences with a wearable multisensor in an outpatient glucose monitoring study, part I: the users' view}

Caduff $A^{l}$, Zanon $M^{1}$, Zakharov $P^{1}$, Mueller $M^{1}$, Talary $M^{1}$, Krebs $A^{1}$, Stahel $W A^{2}$, Donath $M^{3}$

${ }^{1}$ Biovotion AG, Zurich, Switzerland; ${ }^{2}$ Seminar for Statistics, ETH Zurich, Zurich, Switzerland; ${ }^{3}$ Clinic for Endocrinology and Diabetes, University Hospital Basel, Basel, Switzerland

J Diabetes Sci Technol 2018; 12: 562-568 


\section{Background}

In many past studies, it was demonstrated that the use of noninvasive continuous glucose monitoring with a wearable Multisensor device yields helpful data about glucose trends that can enhance diabetes management in controlled and semicontrolled conditions.

\section{Methods}

Twenty subjects with type 1 diabetes participated in a longitudinal study aimed at testing past results in uncontrolled in-clinic and outpatient conditions and to collect Multisensor glucose information. A total of 1072 study days were collected, and a fully online compatible algorithmic routine was applied, linking Multisensor and glucose data to noninvasively evaluate glucose trends. The subjects were asked to run a digital log book, daily semiautomated data transfer, and at least 10 daily self-monitoring of blood glucose $(\mathrm{SMBG})$ values

\section{Results}

The Multisensor was able to present glucose trends either accurately or with one level of error in 9 out of 10 cases. This implies that the Multisensor shows the glucose dynamic to rapidly increase, or at least increase, in $90 \%$ of all cases.

\section{Conclusions}

The Multisensor device and algorithmic routine as utilized under controlled conditions can trace glucose trends in all patients, and in uncontrolled conditions. Although the results are promising, the burden on patients was still quite significant, and future development should employ further automation to reduce it. The component of glucose trend indication was welcomed and very much appreciated by patients and helps justify the use of a wearable sensor device.

\section{First experiences with a wearable multisensor device in a noninvasive continuous glucose monitoring study at home, part II: the investigators' view}

Zanon $M^{l}$, Mueller $M^{l}$, Zakharov $P^{l}$, Talary $M S^{l}$, Donath $M^{2}$, Stahel $W A^{3}$, Caduff $A^{1}$

${ }^{1}$ Biovotion AG, Zurich, Switzerland; ${ }^{2}$ Clinic for Endocrinology and Diabetes, University Hospital Basel, Basel, Switzerland; ${ }^{3}$ Seminar for Statistics, ETH Zurich, Zurich, Switzerland

\section{J Diabetes Sci Technol 2018; 12: 554-561}

\section{Background}

A number of previous studies have demonstrated that the use of noninvasive continuous glucose monitoring with a Multisensor device worn on the upper arm gives helpful data about glucose trends to enhance diabetes management in controlled and semicontrolled conditions.

\section{Methods}

Another study aimed at evaluating the previous findings also in uncontrolled conditions was conducted at home with 20 subjects with type 1 diabetes participating. In all, 1072 study days were collected, and a fully online compatible algorithmic routine was applied, which linked Multisensor data to glucose to noninvasively evaluate glucose trends.

\section{Results}

Glucose values from sensor data were calculated by the algorithm and a daily calibration added a constant. This provided point inaccuracy measured by a mean absolute relative difference of $35.4 \mathrm{mg} / \mathrm{dL}$ on test data. This inaccuracy was higher than current state-of-the-art minimally invasive devices, but $86.9 \%$ of glucose rate points still fall within the zone $A_{R}+B_{R}$, i.e., an evolution of CEG developed for CGM sensors is the rate error grid (REG) that measures the accuracy of estimated glucose trends with analogous regions labeled from AR to ER.

\section{Conclusions}

The Multisensor device and the algorithmic routine previously used earlier in controlled conditions also tracked glucose changes in uncontrolled conditions, yet with lower accuracy. The investigation of the learning curves suggests that obtaining more data would not improve the results. It is therefore important that further efforts should focus on the development of more complex algorithmic routines.

\section{Comments}

The importance of these two complementary articles is that they devote an equal amount of reference to the investigators' view and the participants' view (e.g., user experience, trend information, and patient observations) regarding the use of first experiences with a wearable Multisensor in outpatient glucose monitoring conditions. The authors conclude that today, users are requesting "wearables" that provide multiple features and preferably in a first step also offer the capability to follow glucose trends and, if possible, do not require calibration. At first glance it seems that these two areas are completely different. Users are mainly interested in convenience issues such as upper arm attachment (length and tightness) or sweating while investigators are interested in improving accuracy. However, a closer examination shows that the perspective of the user and the researcher are interacting and influencing each other. For example, a band that is too long led to inappropriate attachment and reduction of data quality in the dielectric sensors. On the other hand, the authors conclude that understanding possible directions for improvement is essential to shrink the accuracy gap and provide a noninvasive technological solution that can be used to improve diabetes therapy. In reality, both participants and investigators seek the same outcome: improved glycemic outcomes with reduced diabetes burden.

\section{In-home nighttime predictive low glucose suspend experience in children and adults with type 1 diabetes}

Messer $L H^{1}$, Calhoun $P^{2}$, Buckingham $B^{3}$, Wilson $\mathrm{DM}^{3}$, Hramiak $I^{4}$, Ly $T^{3}{ }^{3}$, Driscoll $M^{5}$, Clinton $P^{3}$, Maahs DM ${ }^{1}$; for the In Home Closed Loop Study Group 
${ }^{I}$ Barbara Davis Center for Childhood Diabetes, University of Colorado Denver, Aurora, CO; ${ }^{2}$ Jaeb Center for Health Research, Tampa, FL; ${ }^{3}$ Division of Endocrinology and Diabetes, Stanford School of Medicine, Palo Alto, CA; ${ }^{4}$ Division of Endocrinology and Metabolism, St Joseph's Health Care, London, ON, Canada; ${ }^{5}$ Diabetes Clinical Trials Unit, St. Joseph's Health Care London, London, ON, Canada

Pediatr Diabetes 2017; 18: 332-339

\section{Background}

Overnight predictive low-glucose suspend (PLGS) decreases hypoglycemia across all ages; be that as it may, there are no reports on conduct or experience differences across age groups, particularly in children and adolescents.

\section{Methods}

One hundred and twenty-seven subjects 4-45 years of age (50\% male) participated in a run-in for a subsequent randomized clinical trial (RCT), using experimental PLGS system nightly for 5-10 nights (PLGS active phase). Number of blood glucose (BG) checks and boluses given per age group were evaluated. In a subsequent 42-night RCT phase, the investigators evaluated sensor use, skin reactions, errors, and reasons why the experimental system was not used.

\section{Results}

In 821 nights of active PLGS, 4- to 6-year-old subjects (and their parents) tested BG levels on $75 \%$ of nights vs $65 \%$ of nights for those 7-10 years old, 53\% of nights for 11-14 year olds, $33 \%$ of nights for $15-25$ year olds, and $28 \%$ of nights for $26-45$ year olds, $(P<0.001)$. Additionally, during active PLGS use, the youngest subjects (and parents) used insulin boluses $56 \%$ of nights, compared with $48 \%$, 33\%, $20 \%$, and $25 \%$, respectively, for the other age groups $(P<0.001)$. Throughout the RCT phase, 4 - to 6-year-old subjects exhibited more frequent and severe skin reactions $(P=0.02)$, while adult subjects (26-45 years old) wore individual sensors a median of 26 hours longer than the youngest subjects $(P<0.001)$. Technical problems with the sensors, such as errors, miscalibrations, etc., as well as traveling and high bedtime BG levels ( $>270$ ), were major contributors to nonuse of the system.

\section{Conclusions}

It is important to explore and understand the diverse utilization patterns and challenges in children, adolescents, and across all age ranges in order to guide patient education to optimize the use of PLGS and future artificial pancreas systems.

\section{Comments}

The progress made in the development of artificial pancreatic systems and the transition from the field of research into the applied and commercial fields make it clear that these systems are technically feasible. It is, however, much less certain that they will be widely adopted by clinicians and patients. In addition, it is clear that there is not a singular AP. Rather, these systems will evolve over time to increase in automation, increase in sophistication, and increase in their ability to normalize blood glucose levels (6) The overnight PLGS is a technological milestone in the AP pathway. Although apparently safe and effective at reducing nocturnal hypoglycemia, sufficient acknowledgment or support for the usability of the system remains a critically neglected aspect, especially in pediatrics (the population with the highest prevalence of severe hypoglycemia). This important study reports on the experience and patient behaviors (BG checking and bolusing) of children, their parents, and young adults during active PLGS use. Future studies must continue to clarify behavioral factors important to children and their parents on AP iterations in order to inform improved provider approaches to education and expectations.

\section{Optimizing hybrid closed-loop therapy in adolescents and emerging adults using the MiniMed 670G system}

Messer $L H^{1}$, Forlenza $G P^{l}$, Sherr $J L^{2}$, Wadwa $R P^{l}$, Buckingham $B A^{3}$, Weinzimer $S A^{2}$, Maahs $D M^{3}$, Slover $R H^{I}$

${ }^{I}$ Barbara Davis Center for Childhood Diabetes, University of Colorado, Anschutz Medical Campus, Aurora, CO; ${ }^{2}$ Department of Pediatrics, Yale School of Medicine, New Haven, CT; ${ }^{3}$ Department of Pediatrics, Stanford University School of Medicine, Stanford, CA

Diabetes Care 2018; 41: 789-796

This manuscript is also discussed in the article on Diabetes Technology and Therapy in the Pediatric Age Group, page S-123.

\section{Objective}

MiniMed 670G is the first commercial hybrid closed-loop (HCL) system for managing type 1 diabetes. The authors collected information from adolescent and young adult subjects and compared insulin delivery patterns and time-inrange metrics in HCL (Auto Mode) and open-loop (OL). System alerts, utilization profiles, and operational parameters were evaluated in order to give clinicians recommendations on how they can optimize clinical use of the system in treating T1D.

\section{Research and Methods}

Thirty-one adolescents and emerging adults (14-26 years of age) from three clinical sites participated in the study. A 2week run-in period in OL was followed by a 3-month in-home study period during which HCL functionality was enabled. Information between baseline OL and HCL use was compared after 1 week, 1 month, 2 months, and 3 months.

\section{Results}

Carbohydrate-to-insulin (C:I) ratios were more aggressive for all meals with HCL compared with baseline OL. 
Daily insulin dosage and basal-to-bolus proportion were unchanged. After 3 months using Auto Mode, time in range increased $14 \%(P<0.001)$, and HbA1c decreased $0.75 \%$. The main reasons for Auto Mode exits were sensor/insulin delivery alerts and hyperglycemia. The percentage of time in Auto Mode gradually declined by $15 \%$, from $87 \%$ to a final use rate of $72 \%$.

\section{Conclusions}

When transitioning young patients to the $670 \mathrm{G}$ system, providers should expect immediate C:I ratio adjustments along with evaluating active insulin time. Users ought to envision occasional Auto Mode exits and should understand that these can be reduced by following system instructions and reliably bolusing for meals. Care providers should render ongoing clinical guidance and education for the use of the system.

\section{Comments}

The MiniMed 670G System from Medtronic is the first commercially available HCL system. The system uses a proprietary proportional-integral-derivative controller with insulin feedback to calculate insulin doses continually based on CGM levels $(7,8,9)$. As AP systems are taken out of the laboratory and utilized in clinical settings, it is important to remember that the role of the human factor in the effective use of AP remains central. The authors of this study underscore some important teaching points learned from the trial that may be useful in implementing $670 \mathrm{G}$ into clinical care concerning the human factor. The importance of education (e.g., expecting system to optimize over a period of days, not hours, or reducing direct exits from Auto Mode due to hyperglycemia by changing C:I ratios and active insulin time and decreasing the number of missed meal boluses) and matching patients' expectations (e.g., patient can expect to be in Auto Mode most of the time if wearing CGM sensor consistently but will still revert to OL 20$30 \%$ of the time) are taking a central place in thinking about improving product efficiency. Realistic expectations of device functionality and users' interactions with it are essential for both health-care professionals and users. Early experiences show a steep learning curve in terms of managing the demands of new technologies, and effective support through these early days is essential for optimal outcomes.

\section{Real-time continuous glucose monitoring facilitates feelings of safety in older adults with type 1 diabetes: a qualitative study}

Litchman ML, Allen NA

University of Utah College of Nursing, Salt Lake City, UT

\section{J Diabetes Sci Technol 2017; 11: 988-995}

\section{Background}

Older adults with type 1 diabetes (T1D) are at higher risk for hypoglycemia, which can lead to cardiovascular and cerebrovascular events, falls, seizures, and hospitalizations.
Real-time continuous glucose monitoring (RT-CGM) helps improve diabetes management and well-being in patients of all ages. Yet, not much is known about older adults and RTCGM use. The aim of this study was to evaluate the benefits of RT-CGM use by individuals with T1D aged 65 years or above.

\section{Methods}

A convenience sample of older adults with T1D completed one of two online surveys regarding RT-CGM. The first survey questioned individuals who were current RT-CGM users, and the second survey, occurring 1 month later, examined individuals who were not currently using RT-CGM but had a desire to do so. Categorical information was analyzed using descriptive statistics. A qualitative content analysis was conducted for open-ended answers.

\section{Results}

Twenty-two subjects were included in this study, of which $50 \%$ were utilizing RT-CGM and 50\% were not but wanted to do so. Three central themes were identified: (i) RT-CGM enhances feelings of safety by preventing hypoglycemia, (ii) RT-CGM enhances well-being, and (iii) access is an obstacle to RT-CGM utilization.

\section{Conclusions}

RT-CGM was proved to enhance safety and well-being in older adults with T1D by preventing hypoglycemia and related injury and worry. Older adults without access to RTCGM experienced more severe hypoglycemia events that caused adverse influences on their safety and well-being. Caregivers of older T1D patients should increase the access to RT-CGM in order to improve their health and safety.

\section{Comments}

One of the most important challenges in diabetes technologies from the human factor perspective is understanding the suitability of devices for optimal use in different age groups. This is especially important among the elderly population. Older people are at increased risk for severe hypoglycemia (due to reduced awareness of hypoglycemic warning symptoms, reduced counterregulatory response, and altered psychomotor performance) and may benefit greatly from the use of a continuous glucose monitor. Although older adults report a willingness to adopt technology usage, data suggest that older adults are part of the "digital divide" (10), a distinction made between those who adopt technology and those who are unable to do so. However, this blanket term hides an ability and willingness by older adults to embrace technologies, and they should not be denied access on the basis of age. More recent surveys in the United States show that in 2009 computer and Internet use among those aged $\geq 65$ years has increased to about $40 \%$ (11) and continues to increase year on year. The current study presents encouraging data in this regard. The authors conclude that the results indicate that RT-CGM is a well-accepted technology that improves perceived safety and well-being in older adults. 
Schooling diabetes: use of continuous glucose monitoring and remote monitors in the home and school settings

Erie $C^{1}$, Van Name $M A^{2}$, Weyman $K^{2}$, Weinzimer $S A^{2}$, Finnegan $J^{2}$, Sikes $K^{2}$, Tamborlane $W V^{2}$, Sherr $J L^{2}$

${ }^{I}$ Charles E. Schmidt College of Medicine, Florida Atlantic University, Boca Raton, FL; ${ }^{2}$ Department of Pediatrics, Yale School of Medicine, New Haven, CT

\section{Pediatr Diabetes 2018; 19: 92-97}

This manuscript is also discussed in the article on Practical Implementation of Diabetes Technology: It Is Time, page S-148.

\section{Background}

Management of T1D has been improved immensely in the past few years, yet many pediatric patients still struggle to achieve optimal glycemic control. It is hoped that with continuous glucose monitoring (CGM) with remote access, this could be overcome, when parents will be able to monitor glucose, even when the child is not with them.

\section{Objective}

The aim of this study was to examine the use of real-time and remote CGM routines by parents and daytime caregivers in homes and schools and to evaluate their expectations of such devices.

\section{Methods}

Fifty participants (33 parents and 17 daytime caregivers) completed an anonymous survey evaluating features of CGM use. Cross-sectional information was collected and the data were analyzed using quantitative and qualitative methods.

\section{Results}

Threshold alerts were utilized most often, followed by rate of change alerts. Most parents and daytime caregivers responded to low- and high-threshold CGM alerts by affirming with a glucose meter prior to treatment, but approximately one-third supported a practice of treating lows without a testing to confirm. Most parents expected their child's daytime caregiver to respond to CGM alerts, and daytime caregivers felt the parent's expectations of them were acceptable. All parents as well as most caregivers reported reduced worry and stress when using CGM.

\section{Conclusions}

The positive attitude of the parents and caregivers to the use of CGM with remote monitoring should promote the use of the CGM in the treatment of children with T1D both at home and in school.

\section{Comments}

In a recent report (12) on the use of continuous glucose in children, it was found that the frequency of SMBG was highest, while CGM use was lowest in pediatric patients in comparison with other age groups. However, more technology over the past few years, including longer wear time, improved accuracy, and remote monitoring capabilities, have resulted in a 2- to 3-fold increase in CGM use in preschool and school-aged patients and consequently led to incorporation of these devices into the classroom environment. Managing diabetes in preschool and school-aged children can be a major challenge as well as an emotional burden for parents and teachers at school. Because parents and teachers play a central role in monitoring and managing diabetes, it is necessary to understand the real-time and remote CGM practices at home and in schools, attitudes regarding its use, and the expectations of parents and caregivers in using this diabetes management tool. This information is critical, as diabetes technology will continue to evolve in the coming years and use of these devices will undoubtedly increase. The authors found that the use of real-time monitoring at school was a common reason parents initiated this technology, with parents noting that CGM with remote monitoring would benefit both themselves and school nurses. The technology helped to address concerns about school and concerns about hypos at night, which remains a major concern for many parents. Other important benefits were real-time alerts and detection of blood glucose trends. The ability to share care remotely between parents and caregivers, based on real-time data, is particularly beneficial and reassuring to both parties. This article reports an important aspect of shared care in the context of CGM and its potential to relieve burden as well as improve glycemic control.

\section{Continuous glucose monitoring in patients with type 1 diabetes and impaired awareness of hypoglycemia: also effective in patients with psychological distress?}

van Beers $C A J^{1}$, de Wit $M^{2}$, Kleijer $S J^{1}$,

Geelhoed-Duijvestijn $\mathrm{PH}^{3}$, DeVries $\mathrm{JH}^{4}$, Kramer $\mathrm{MHH}^{1}$, Diamant $M^{1}$, Serné $E H^{1}$, Snoek $F J^{2,5}$

Departments of ${ }^{1}$ Internal Medicine and ${ }^{2}$ Medical Psychology, VU University Medical Center, Amsterdam, The Netherlands; ${ }^{3}$ Department of Internal Medicine, Medical Center Haaglanden, The Hague, The Netherlands; Departments of ${ }^{4}$ Endocrinology and ${ }^{5}$ Medical Psychology, Academic Medical Center, University of Amsterdam, Amsterdam, The Netherlands

\section{Diabetes Technol Ther 2017; 19: 595-599}

\section{Background}

The goal of this research was to assess whether psychological distress modifies the effect of continuous glucose monitoring (CGM) in patients with T1D and impaired awareness of hypoglycemia.

\section{Methods}

Fifty-two subjects with T1D and impaired awareness of hypoglycemia took part in a previously reported randomized 
crossover trial with two 16-week intervention periods comparing CGM with self-monitoring of blood glucose (SMBG).

\section{Results}

Amid the CGM phase, time spent in euglycemia (4$10 \mathrm{mmol} / \mathrm{L}$ ), the primary outcome, was $9.6 \%$ higher compared with the SMBG phase $(P<0.0001)$. Psychological distress was operationalized as low emotional well-being (World Health Organization Well-Being Index $5<50$ ), high diabetes-related distress (Problem Areas in Diabetes $5 \geq 8$ ), and/or high fear of hypoglycemia (Hypoglycemia Fear Survey [HFS] Worry $>$ mean HFS Worry score +1 standard deviation). Modifying effects were evaluated by analyzing psychological distress score $\times$ intervention-interaction effects. Results showed that both the low emotional well-being group and normal emotional well-being group had equal glycemic results during the CGM phase. High diabetes distress and increased fear of hypoglycemia did not result in significant interaction effects for glycemic outcomes.

\section{Conclusions}

This research showed that CGM is equally effective in terms of glycemic improvements in high versus low distressed participants with T1D and impaired awareness of hypoglycemia.

\section{Comments}

The results of this study are encouraging, considering that previous studies have reported that with CGM, patients are faced with real-time feedback on blood glucose variation and alarms that may be experienced as stressful and difficult to handle $(13,14)$. It seemed reasonable to assume that those patients with preexisting high levels of distress (e.g., low emotional well-being, high diabetesrelated distress, and fear of hypoglycemia) would achieve poorer results using CGM. Surprisingly, it was found that the effect of CGM on the primary outcome (time spent in euglycemia) remained significant also in those with low emotional well-being. Furthermore, the lower effect sizes were mainly due to better glycemic control during the SMBG phase rather than poorer glycemic control during the CGM phase. The results of the study corroborate the findings of another study recently published by Giani et al. and found that "consistent and durable CGM use in youth with T1D is associated with treatment adherence and improved glycemic control without increasing psychosocial distress" (15). Further, it demonstrates that those with high levels of distress should not be excluded from access to CGM; rather, an open mind should be maintained and CGM discussed as an option.

\section{Author Disclosure Statement}

No competing financial interests exist.

\section{References}

1. Scaramuzza A, Iafusco D, Lombardo F, et al. Adolescent use of insulin and patient-controlled analgesia pump technology: a 10-year food and drug administration retrospective study of adverse events. Pediatrics 2008; 122: 473-474, author reply 4.

2. Cope JU, Samuels-Reid JH, Morrison AE. Pediatric use of insulin pump technology: a retrospective study of adverse events in children ages 1-12 years. J Diabetes Sci Technol 2012; 6: 1053-1059.

3. Barnard K, Parkin C, Young A, et al. Use of an automated bolus calculator reduces fear of hypoglycemia and improves confidence in dosage accuracy in patients with type 1 diabetes mellitus treated with multiple daily insulin injections. J Diabetes Sci Technol 2012; 6: 144-149.

4. Schmidt S, Meldgaard M, Serifovski N, et al. Use of an automated bolus calculator in MDI-treated type 1 diabetes: the BolusCal Study, a randomized controlled pilot study. Diabetes Care 2012; 35: 984-990.

5. Ziegler R, Cavan DA, Cranston I, et al. Use of an insulin bolus advisor improves glycemic control in multiple daily insulin injection (MDI) therapy in patients with suboptimal glycemic control. First results from the ABACUS trial. Diabetes Care 2013; 36: 3613-3619.

6. Kowalski A. Pathway to artificial pancreas systems revisited: moving downstream. Diabetes Care 2015; 38: 1036-1043.

7. Ruiz JL, Sherr JL, Cengiz E, et al. Effect of insulin feedback on closed-loop glucose control: a crossover study. $J$ Diabetes Sci Technol 2012; 6: 1123- 1130.

8. Steil GM. Algorithms for a closed-loop artificial pancreas: the case for proportional-integral derivative control. $J$ Diabetes Sci Technol 2013; 7: 1621-1631.

9. Weinzimer SA, Steil GM, Swan KL, Dziura J, Kurtz N, Tamborlane WV. Fully automated closed-loop insulin delivery versus semiautomated hybrid control in pediatric patients with type 1 diabetes using an artificial pancreas. Diabetes Care 2008; 31: 934-939.

10. National Telecommunications and Information Administration. A nation online: entering the broadband age. Washington, DC: U.S. Department of Commerce, 2004. Available at www.ntia.doc.gov/reports/anol/NationOnline Broadband04.htm (Accessed June 2, 2010).

11. Mitzner TL, Boron JB, Fausset CB, et al. Older adults talk technology: technology usage and attitudes. Comput $\mathrm{Hu}$ man Behav 2010; 26: 1710-1721.

12. Miller KM, Foster NC, Beck RW, et al. Current state of type 1 diabetes treatment in the U.S.: updated data from the T1D Exchange Clinic Registry. Diabetes Care 2015; 38: 971-978.

13. Tanenbaum ML, Hanes SJ, Miller KM, et al. Diabetes device use in adults with type 1 diabetes: barriers to uptake and potential intervention targets. Diabetes Care 2017; 40: 181-187.

14. Pickup JC, Ford HM, Samsi K. Real-time continuous glucose monitoring in type 1 diabetes: a qualitative framework analysis of patient narratives. Diabetes Care 2015; 38: 544-550.

15. Giani E, Snelgrove R, Volkening LK, Laffel LM. Continuous glucose monitoring (CGM) adherence in youth with type 1 diabetes: associations with biomedical and psychosocial variables. J Diabetes Sci Technol 2017; 11: 476-483. 